POS PROCEEDINGS

\title{
First SND detector results on hadron cross sections at VEPP-2000 $e^{+} e^{-}-$collider
}

Dimova T.V. ${ }^{* \dagger}$

Budker Institute of Nuclear Physics, Russia

M.N.Achasov, A.Yu.Barnyakov, K.I.Beloborodov, A.V.Berdyugin, A.G.Bogdanchikov, A.A.Botov, D.A.Bukin, V.B.Golubev, K.A.Grevtsov, V.P.Druzhinin, A.S.Kasaev, L.V.Kardapoltsev, A.G.Kharlamov, I.A.Koop, D.P.Kovrizhin, A.A.Korol, S.V.Koshuba, A.P.Lysenko, K.A.Martin, A.E.Obrazovsky, E.V.Pakhtusova, Yu.A.Rogovsky, A.L.Romanov, S.I.Serednyakov, P.Yu.Shatunov, Yu.M.Shatunov, D.A.Shtol, D.B.Shwartz, Z.K.Silagadze, K.Yu.Skovpen, A.N.Skrinsky, I.K.Surin, Yu.A.Tikhonov, A.V.Vasiljev

Budker Institute of Nuclear Physics, Russia

Novosibirsk State University, Russia

The results of the first data-taking runs in the 1-2 GeV range with SND detector at VEPP$2000 e^{+} e^{-}$collider are presented. The reported data correspond to the integrated luminosity of about $7.5 \mathrm{pb}^{-1}$. The cross sections of the multihadron processes $e^{+} e^{-} \rightarrow$ $\pi^{+} \pi^{-} \pi^{0}, \pi^{0} \pi^{0} \gamma, \pi^{+} \pi^{-} \pi^{0} \pi^{0}, \eta \pi^{+} \pi^{-}$, and nucleon-antinucleon pair production are measured.

The 2011 Europhysics Conference on High Energy Physics, EPS-HEP 2011,

July 21-27, 2011

Grenoble, Rhône-Alpes, France

*Speaker.

†baiert@inp.nsk.su 


\section{Introduction}

The SND detector [1] is the general purpose nonmagnetic detector for low energy $e^{+} e^{-}$colliders. It consists of the drift-chamber tracking system, the aerogel Cherenkov counters, the three-layer spherical electromagnetic calorimeter with $1680 \mathrm{NaI}(\mathrm{Tl})$ crystals, and the muon detector with plastic scintillator counters and proportional tubes.

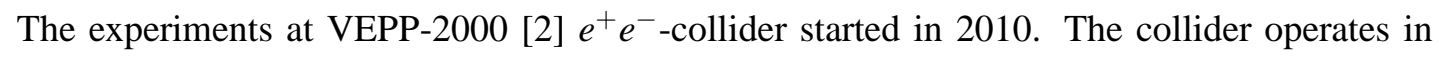
the c.m. energy range from 2 pion threshold up to $2 \mathrm{GeV}$. In 2010 and 2011 two scans in the energy range $2 E=1.0-2.0 \mathrm{GeV}$ (MHAD2010 and MHAD2011) were performed with the integrated luminosity of 5 and $25 \mathrm{pb}^{-1}$, respectively [B] (Table 『).

\begin{tabular}{|c|c|c|c|}
\hline Experiment & Energy region $(\mathrm{GeV})$ & Energy step $(\mathrm{MeV})$ & Luminosity $\left(\mathrm{pb}^{-1}\right)$ \\
\hline MHAD2010 & $1.1 \div 1.9$ & 100 & 5 \\
MHAD2011 & $1.05 \div 2.0$ & 25 & 25 \\
\hline
\end{tabular}

The achieved luminosity is $0.8 \cdot 10^{31} \mathrm{~cm}^{-2} \mathrm{~s}^{-1}$ at the $2 E=1 \mathrm{GeV}$ and $2 \cdot 10^{31} \mathrm{~cm}^{-2} \mathrm{~s}^{-1}$ at the $2 E=2 \mathrm{GeV}$.

SND physical program covers measurements of total and different exclusive hadronic cross sections, study of the excited vector mesons $\rho^{\prime}, \omega^{\prime}, \phi^{\prime}$ etc, and study of nucleon-antinucleon pair production $e^{+} e^{-} \rightarrow n \bar{n}, p \bar{p}$ near threshold.

\section{Multihadron cross sections}

The multihadron cross sections have been measured on the MHAD2010 data. The process $e^{+} e^{-} \rightarrow \omega \pi^{0}$ is studied in the $\pi^{0} \pi^{0} \gamma$ final state. The measured cross section in comparison with the results of previous experiments [4, [5, 6] is shown in Fig. [1]. The fit is done by a the sum of $\rho(770)$ and $\rho(1450)$ contributions. For luminosity normalization the process $e^{+} e^{-} \rightarrow \gamma \gamma$ is used. The SND data and the "CLEO data" obtained from spectral function in $\tau \rightarrow \omega \pi \nu$ decay using CVC are in reasonable agreement.

The process $e^{+} e^{-} \rightarrow \pi^{+} \pi^{-} \pi^{0} \pi^{0}$ dominates in the energy region between 1 and $2 \mathrm{GeV}$. The measured cross section is shown in Fig. 1 $1 \mathrm{~b}$. The main contributions come from the $\rho(1450)$ and $\rho(1700)$ decays. The measured cross section is in good agreement with the previous SND data (below $1.4 \mathrm{GeV}$ ) [8] and the BABAR data obtained using ISR method [10].

In the $e^{+} e^{-} \rightarrow \pi^{+} \pi^{-} \pi^{0}$ cross section (Fig. 1 $\mathrm{k}$ ) the $\omega(1420)$ and $\omega(1650)$ are seen. Our result is in reasonable agreement with the previous SND [12] and BABAR [13] measurements.

The cross section of $e^{+} e^{-} \rightarrow \eta \pi^{+} \pi^{-}$(Fig. 1 $1 \mathrm{~d}$ ) is measured in $\eta \rightarrow 2 \gamma$ decay mode. It is dominated by the $\rho(1450) \rightarrow \eta \pi^{+} \pi^{-}$decay. The measured cross section agrees with the previous SND result [14] as well as the results from other experiments [15] 16].

\section{Nucleon-antinucleon processes}

The $e^{+} e^{-} \rightarrow n \bar{n}$ cross section is measured with the part $\left(2.5 \mathrm{pb}^{-1}\right)$ of the MHAD2011 data sample. The events are selected with the efficiency of about $30 \%$. The background from cosmic rays, $e^{+} e^{-} \rightarrow p \bar{p}$, and $e^{+} e^{-} \rightarrow \gamma \gamma$ processes is subtracted. The resulting cross section (Fig.『) is 

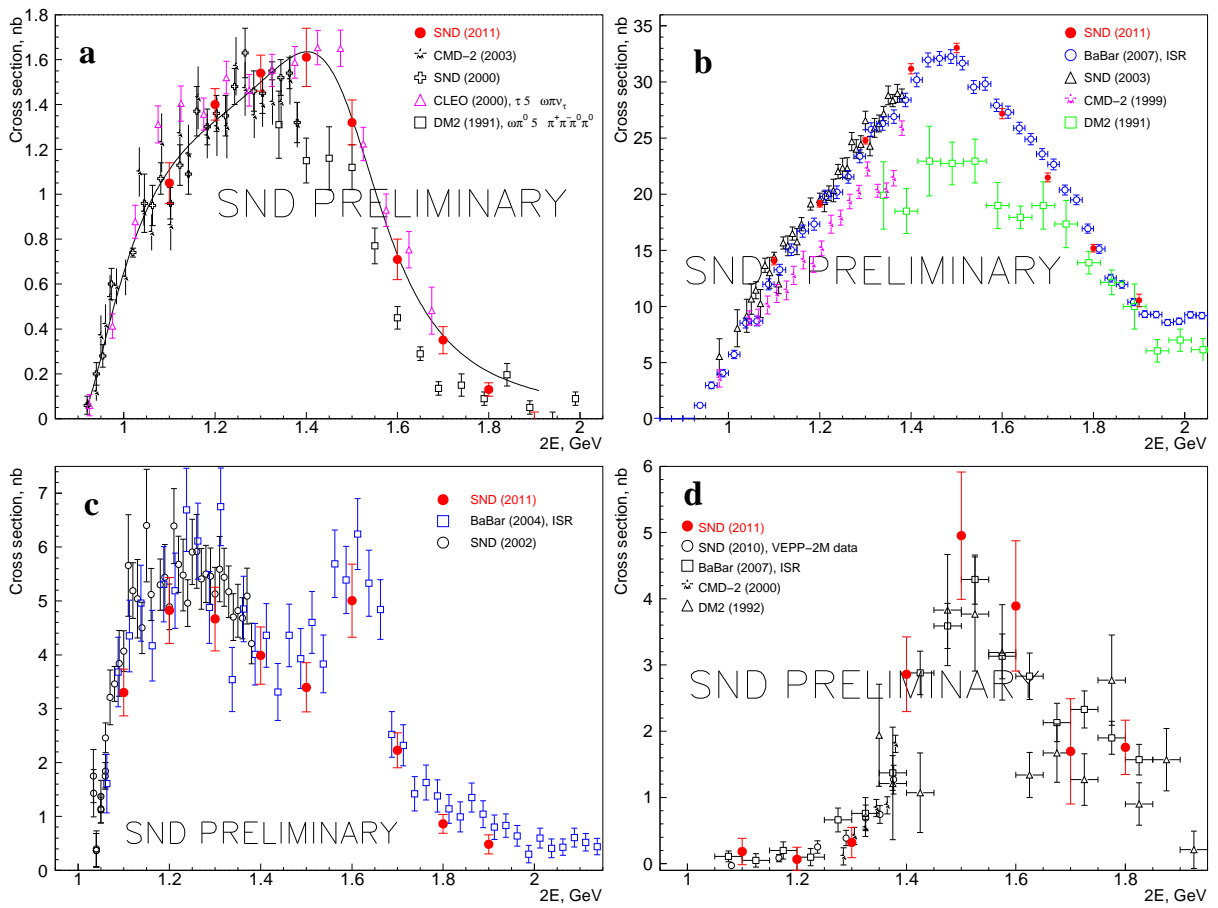

Figure 1: The measured multihadron cross sections in comparison with previous experiments: a) $e^{+} e^{-} \rightarrow$ $\omega \pi^{0} \rightarrow \pi^{0} \pi^{0} \gamma$ [4, 5, 6, 7]; b) $e^{+} e^{-} \rightarrow \pi^{+} \pi^{-} \pi^{0} \pi^{0}$ [8, 9, 10, 11]; c) $e^{+} e^{-} \rightarrow \pi^{+} \pi^{-} \pi^{0}$ [12, 13]; d) $e^{+} e^{-} \rightarrow$ $\eta \pi^{+} \pi^{-}$[14, 15, 16, 17].

fit with a step function. This gives $\sigma=0.1 \pm 0.2 \mathrm{nb}$ below the $n \bar{n}$ threshold and $\sigma=0.8 \pm 0.2 \mathrm{nb}$ above. Our result agrees with FENICE measurement [18]. All quoted errors are statistical. Sources of systematic uncertainties are to be analyzed.

The $e^{+} e^{-} \rightarrow p \bar{p}$ process is studied using about $3 \mathrm{pb}^{-1}$ of the MHAD2011 data. The detection efficiency is about $40 \%$. The background is estimated on data below the $p \bar{p}$ threshold and is found to be about $6 \%$. The measured cross section is shown at Fig. 2 $\mathrm{b}$ in comparison with BABAR data [19].
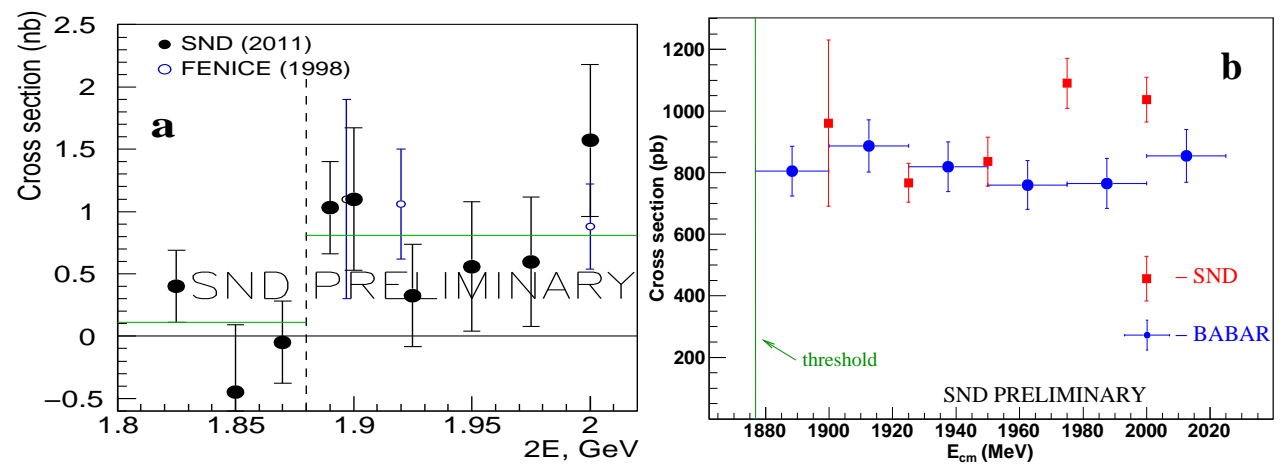

Figure 2: The measured nucleon-antinucleon cross sections in comparison with previous experiments: a) $e^{+} e^{-} \rightarrow n \bar{n}$ [18], b) $e^{+} e^{-} \rightarrow p \bar{p}$ [19]. 


\section{Conclusions}

SND detector began to operate in 2010. During two years data with integrated luminosity about $30 \mathrm{pb}^{-1}$ have been collected. The preliminary results on different hadron processes have been obtained using a part of the collected data. All results are in reasonable agreement with previous measurements. The analysis of the full data sample as well as new experimental run will be carried out in nearest future.

\section{Acknowledgements}

This work is partially supported by the Russian Federation Presidential Grant for Scientific Schools NSh-6943.2010.2, Russian Fund for Basic Research Grant 11-02-00276-a, and the Grant 14.740.11.1167 of Federal Program 'Scientific and Pedagogical Personnel of Innovational Russia”.

\section{References}

[1] M.N. Achasov et al., Nucl. Instrum. Meth. A598 (2009) 31.

V.M. Aulchenko et al., Nucl. Instrum. Meth. A598, (2009) 102.

[2] Yu.M. Shatunov et al., in proceedings of the 7th European Particle Accelerator Conference, Vienna, 2000, p. 439.

[3] P.M.Astigeevich et al., Preprint BINP 2011-21, Novosibirsk, 2011.

[4] M.N.Achasov et al., Phys. Lett. B486 (2000) 20 [hep-ex/0005032].

[5] R.R.Akhmetshin et al., Phys. Lett. B562 (2003) 173 [hep-ex/0304009].

[6] K.W.Edwards et al., Phys. Rev. D61 (2000) 072003 [hep-ex/9908024].

[7] D.Bisello et al., Nucl.Phys.Proc.Suppl. 21 (1991) 111.

[8] M.N.Achasov et al., J. Exp. Theor. Phys. 96 (2003) 789.

[9] R.R.Akhmetshin et al., Phys. Lett. B466 (1999) 392.

[10] V.P.Druzhinin, in proceedings of LP07 International Symposium, (2007) 134 [hep-ex/0710.3455].

[11] Bisello et al., Nucl. Phys. Proc. Suppl. 21 (1991) 111.

[12] M.N.Achasov et al., in proceedings of AIP Conference, 717 (2004) 60 [hep-ex/0309055].

[13] B.Aubert et al., Phys. Rev. D70 (2004) 072004.

[14] M.N.Achasov et al., JETP Lett. 92 (2010) 84.

[15] R.R.Akhmetshin et al., Phys. Lett. B489 (2000) 125.

[16] B.Aubert et al., Phys. Rev. D76 (2007) 092005.

[17] Antonelli et al., Phys. Lett. B212 (1988) 133.

[18] A.Antonelli et al., Nucl. Phys. B517 (1998) 3.

[19] B.Aubert et al., Phys. Rev. D71 (2006) 012005 [hep-ex/0512123]. 